\title{
Multiband $\mathrm{k}$ center dot $\mathrm{p}$ model and fitting scheme for ab initio based electronic structure parameters for wurtzite GaAs
}

\author{
Marquardt, Oliver; Caro, Miguel A.; Koprucki, Thomas; Mathe, Peter; Willatzen, Morten
}

Published in:

Physical Review B

Link to article, DOI:

10.1103/PhysRevB.101.235147

Publication date:

2020

Document Version

Publisher's PDF, also known as Version of record

Link back to DTU Orbit

Citation (APA):

Marquardt, O., Caro, M. A., Koprucki, T., Mathe, P., \& Willatzen, M. (2020). Multiband k center dot p model and fitting scheme for ab initio based electronic structure parameters for wurtzite GaAs. Physical Review B, 101(23), [235147]. https://doi.org/10.1103/PhysRevB.101.235147

\section{General rights}

Copyright and moral rights for the publications made accessible in the public portal are retained by the authors and/or other copyright owners and it is a condition of accessing publications that users recognise and abide by the legal requirements associated with these rights.

- Users may download and print one copy of any publication from the public portal for the purpose of private study or research.

- You may not further distribute the material or use it for any profit-making activity or commercial gain

- You may freely distribute the URL identifying the publication in the public portal 


\title{
Multiband $\mathbf{k} \cdot \mathbf{p}$ model and fitting scheme for $a b$ initio based electronic structure parameters for wurtzite GaAs
}

\author{
Oliver Marquardt, ${ }^{1, *}$ Miguel A. Caro $\odot,{ }^{2,3}$ Thomas Koprucki $\odot,{ }^{1}$ Peter Mathé $\odot,{ }^{1}$ and Morten Willatzen $\oplus^{4,5}$ \\ ${ }^{1}$ Weierstraß-Institut für angewandte Analysis und Stochastik, Mohrenstr. 39, D - 10117 Berlin, Germany \\ ${ }^{2}$ Department of Electrical Engineering and Automation, Aalto University, 02150 Espoo, Finland \\ ${ }^{3}$ Department of Applied Physics, Aalto University, 02150 Espoo, Finland \\ ${ }^{4}$ Department of Photonics Engineering, Technical University of Denmark, DK-2800 Kongens Lyngby, Denmark \\ ${ }^{5}$ Beijing Institute of Nanoenergy and Nanosystems, Chinese Academy of Sciences, Beijing 100083, China
}

(Received 5 March 2020; revised manuscript received 15 May 2020; accepted 11 June 2020; published 22 June 2020)

\begin{abstract}
We develop a 16-band $\mathbf{k} \cdot \mathbf{p}$ model for the description of wurtzite GaAs, together with a scheme to determine electronic structure parameters for multiband $\mathbf{k} \cdot \mathbf{p}$ models. Our approach uses low-discrepancy sequences to fit $\mathbf{k} \cdot \mathbf{p}$ band structures beyond the eight-band scheme to most recent $a b$ initio data, obtained within the framework for hybrid-functional density functional theory with a screened-exchange hybrid functional. We report structural parameters, elastic constants, band structures along high-symmetry lines, and deformation potentials at the $\Gamma$ point. Based on this, we compute the bulk electronic properties ( $\Gamma$ point energies, effective masses, Luttinger-like parameters, and optical matrix parameters) for a ten-band and a sixteen-band $\mathbf{k} \cdot \mathbf{p}$ model for wurtzite GaAs. Our fitting scheme can assign priorities to both selected bands and $\mathbf{k}$ points that are of particular interest for specific applications. Finally, ellipticity conditions can be taken into account within our fitting scheme in order to make the resulting parameter sets robust against spurious solutions.
\end{abstract}

DOI: 10.1103/PhysRevB.101.235147

\section{INTRODUCTION}

The development of novel electronic devices and light sources requires efficient techniques to model the optoelectronic properties of semiconductor nanostructures. For about two decades now, the six- and eight-band $\mathbf{k} \cdot \mathbf{p}$ formalisms represent the backbone of semiconductor device modeling and have been extensively employed to study semiconductor nanostructures of a wide range of shapes, dimensions, and material compositions [1-6]. These approaches describe the bulk electronic band structure of a material perturbatively, such that it is well reproduced in the vicinity of a selected highsymmetry point within the Brillouin zone (BZ), commonly the zone center $\Gamma$. For the description of heterostructures consisting of different materials or crystal phases, the $\mathbf{k} \cdot \mathbf{p}$ formalism is employed within envelope functions that contain the description of the shape and dimensions of the heterostructure in a continuum picture. An accurate description of the electronic properties of semiconductor nanostructures using $\mathbf{k} \cdot \mathbf{p}$ models can be achieved only if the electronic properties are governed by the band structure around the high-symmetry point selected for the corresponding $\mathbf{k} \cdot \mathbf{p}$ model. The small number of relevant electronic structure parameters required for these well-established $\mathbf{k} \cdot \mathbf{p}$ models: band offsets, energy splittings, electron- and hole effective masses (the latter are commonly translated to Luttinger-like parameters), deformation potentials and optical matrix elements, are widely

*oliver.marquardt@wias-berlin.de available for most III-V [7-9] and many II-VI compound semiconductors $[10,11]$.

On the other hand, the eight-band $\mathbf{k} \cdot \mathbf{p}$ model has shortcomings that, despite its past and recent successes, make it unsuited for a number of material systems and devices: The limitation of the set of basis functions to the top three valence bands and the bottom conduction band with their respective $|\uparrow\rangle$ and $|\downarrow\rangle$ components makes this model a priori unsuited to materials such as GaSb or GaAs in the wurtzite (WZ) phase, where an additional conduction band plays a decisive role [12]. The perturbative approach yields a good description of the electronic band structure around the BZ center, $\mathbf{k}=\mathbf{0}$, whereas the limitation to one conduction band and three valence bands coupled via optical matrix elements cannot resolve all features of the band structure throughout the $\mathrm{BZ}$, so that the description of indirect band gap materials such as $\mathrm{Si}$ or $\mathrm{Ge}$, where the minimum of the conduction band is not at the same $\mathbf{k}$ value as the maximum of the valence band, requires a larger basis, e.g., a 15-band or even 30 -band $\mathbf{k} \cdot \mathbf{p}$ model $[13,14]$.

The existing parameter sets, for instance compiled in the remarkable work by Vurgaftman and coworkers [7] and its update for the III-N semiconductors [8], that provide all essential parameters for most compound semiconductors, are limited to the eight-band model. Furthermore, the application of the eight-band $\mathbf{k} \cdot \mathbf{p}$ formalism and envelope function approach to heterostructures can - under some conditions-find erroneous, so-called spurious solutions that result from a loss of ellipticity of the respective $\mathbf{k} \cdot \mathbf{p}$ Hamiltonian operator [15]. While existing parameter sets have been evaluated for selected 

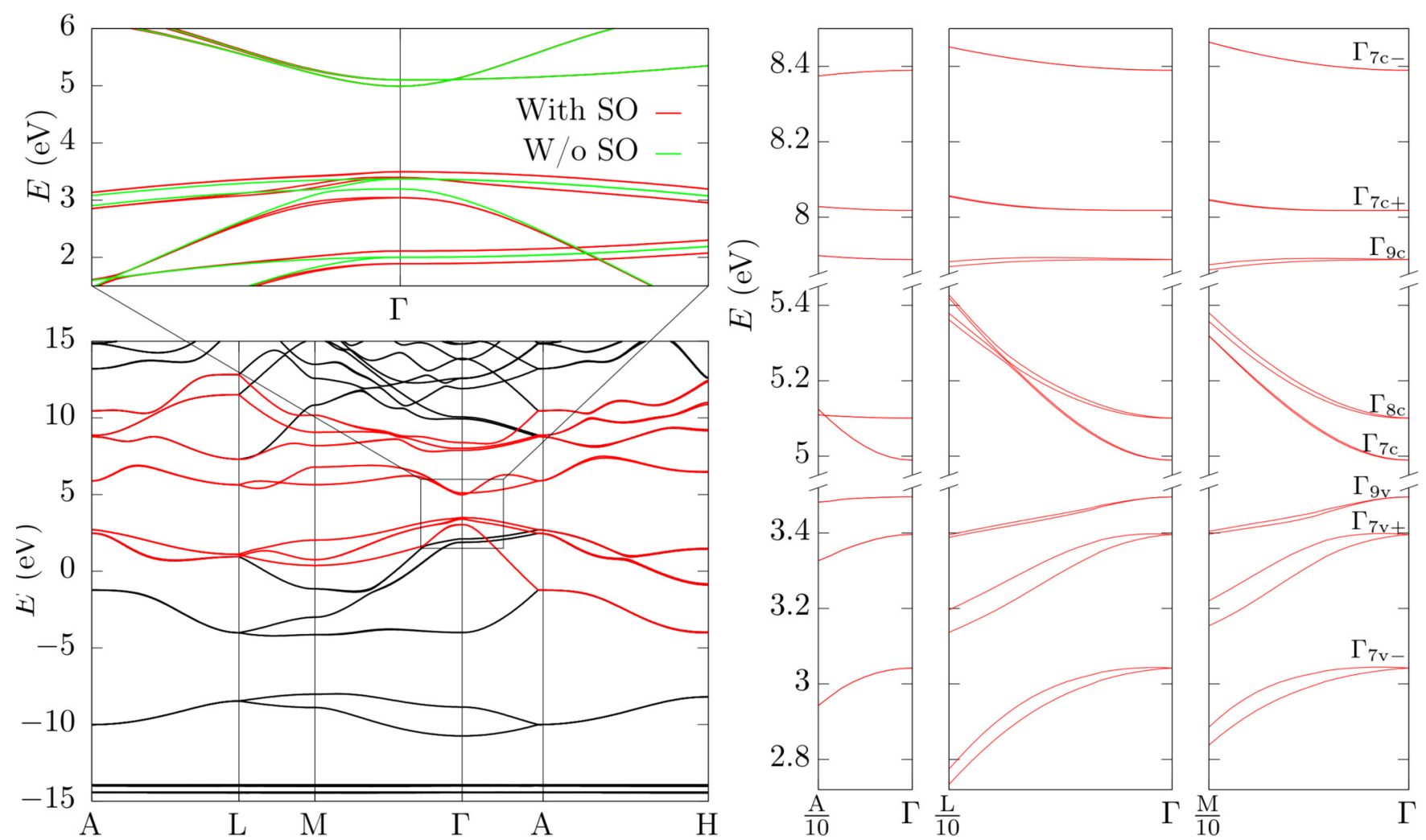

FIG. 1. Left: Band structure for WZ GaAs. Relevant bands for the 16-band Hamiltonian are depicted in red. The vicinity of $\Gamma$ is shown in an enlarged view, comparing simulation with (red) and without (green) SO splitting. Right: Band structure detail around $\Gamma$.

semiconductor materials [15-17], ellipticity conditions have, to our knowledge, never been taken into account directly in the calculation of $\mathbf{k} \cdot \mathbf{p}$ electronic structure parameter sets.

The scope of our work is to provide a versatile tool that can generate parameter sets for $\mathbf{k} \cdot \mathbf{p}$ models of arbitrary complexity and level of sophistication including, but also going beyond, six or eight basis functions. We focus on WZ GaAs, as this material can be employed in novel nanowire-based crystal-phase heterostructures [18] and the description of its electronic structure requires the consideration of at least one additional conduction band $[12,19]$.

We derive a sixteen-band $\mathbf{k} \cdot \mathbf{p}$ Hamiltonian that takes the second relevant conduction band close to the band gap as well as the following three conduction bands into account, to provide a better accuracy and validity of the whole band structure throughout wide parts of the BZ. We compute the corresponding 16-band $\mathbf{k} \cdot \mathbf{p}$ parameter set by fitting the $\mathbf{k} \cdot \mathbf{p}$ band structure to an up-to-date ab initio band structure.

We note that the parameter fitting tool presented is suited to other materials such that it can be employed to provide a full spectrum of up-to-date $\mathbf{k} \cdot \mathbf{p}$ parameters of III-V and II-VI semiconductor materials as well as of any material of which the band structure can be described using a parameterized Hamiltonian, independent of the existence of a band gap. Our approach enables us to increase the fitting priority of selected bands and high-symmetry points within the BZ in order to produce the best-suited set of parameters for applications that rely on the correct description of these bands and highsymmetry points. Furthermore, our tool facilitates the calculation of $\mathbf{k} \cdot \mathbf{p}$ parameter sets that avoid spurious solutions in the simulation of the electronic properties of nanostructures as it allows us to control the nonellipticity of the Hamiltonian operator matrix to a certain extent.

\section{BAND STRUCTURE CALCULATION}

In order to obtain up-to-date band structure parameters for multiband $\mathbf{k} \cdot \mathbf{p}$ models, we have carried out hybrid-functional density functional theory (DFT) calculations of the electronic properties of GaAs in the WZ phase (for details, see Appendix A). Equilibrium lattice parameters and $\mathrm{WZ}$ internal parameter were obtained by fitting the total energy of the crystal to an equation of state. The elastic constants were computed from the stress tensor, rather than total energy derivatives,

TABLE I. Structural and elastic parameters for ZB and WZ GaAs. Experimental values, where available, are in round brackets, values from previous theory in squared brackets. a: Ref. [7], b: Ref. [20], c: Ref. [21].

\begin{tabular}{lcc}
\hline \hline & ZB GaAs & WZ GaAs \\
\hline$a(\AA)$ & $5.671\left(5.642^{\mathrm{a}}\right)$ & $3.997\left(3.989^{\mathrm{b}}\right)$ \\
$c(\AA)$ & $\mathrm{n} / \mathrm{a}$ & $6.588\left(6.564^{\mathrm{b}}\right)$ \\
$u$ & $\mathrm{n} / \mathrm{a}$ & $0.3741\left(0.3746^{\mathrm{c}}\right)$ \\
$C_{11}(\mathrm{GPa})$ & $119\left(122.1^{\mathrm{a}}\right)$ & 142 \\
$C_{12}(\mathrm{GPa})$ & $51\left(56.6^{\mathrm{a}}\right)$ & 46 \\
$C_{13}(\mathrm{GPa})$ & $\mathrm{n} / \mathrm{a}$ & 28 \\
$C_{33}(\mathrm{GPa})$ & $\mathrm{n} / \mathrm{a}$ & 167 \\
$C_{44}(\mathrm{GPa})$ & $80\left(60.0^{\mathrm{a}}\right)$ & 40 \\
\hline \hline
\end{tabular}


TABLE II. Band gaps, position of the topmost valence band with respect to the average electrostatic potential, SO splittings and $\mathrm{CF}$ splittings of WZ GaAs obtained with SO coupling enabled. Values reported in Ref. [34] are given in square brackets for comparison, where applicable.

\begin{tabular}{lc}
\hline \hline$E_{\mathrm{g}}(\mathrm{eV})$ & $1.494[1.453]$ \\
\hline$E_{\mathrm{VB}}(\mathrm{eV})$ & 3.371 \\
$\Delta_{\mathrm{cf}}=\Delta_{1}(\mathrm{w} / \mathrm{o} S O, \mathrm{meV})$ & 177 \\
$\Delta_{\mathrm{cf}}=\Delta_{1}($ with SO, meV) & $184[129]$ \\
$\Delta_{2}=\Delta_{3}=\Delta_{\mathrm{so}} / 3(\mathrm{meV})$ & $123[116]$ \\
\hline \hline
\end{tabular}

which allows for more accurate results [22]. The structural parameters and elastic constants obtained are listed in Table I. Note that the internal WZ parameter $u$ is lower than the ideal value, $u_{\mathrm{id}}=0.375$, unlike for most other WZ materials. This is an indication of inverse polarity (and piezoelectricity) in $\mathrm{GaAs}$, consistent with zincblende (ZB) results, for which the piezoelectric coefficient is of opposite sign when compared to the III nitrides [23]. Our computed values are in good agreement with previously published ones (reference values from experiment or theory are provided in the table).

The band structure of WZ GaAs along high-symmetry lines, with spin-orbit ( $\mathrm{SO}$ ) coupling, is shown in Fig. 1 (left). A detail of the six topmost valence bands and ten lowest conduction bands around the $\Gamma$ point is given in Fig. 1 (right). Basic band structure information is presented in Table II. The WZ $\Delta_{1,2,3}$ parameters have been obtained by fitting Eq. (11) of Chuang and Chang [24] to the energy differences obtained from the DFT calculation. We note that our crystalfield $(\mathrm{CF})$ splitting deviates from a previously reported value, whereas band gap and SO coupling are in good agreement (cf. Tab. II).

The SO coupling Hamiltonian acts as a perturbation, nonself-consistently, on top of the self-consistent DFT band structure [25]. Thus, the effects of strain on the band structure (i.e., the deformation potentials) are well captured in the absence of SO coupling. The $\mathbf{k} \cdot \mathbf{p}$ Hamiltonian also treats SO coupling as a perturbation, with the strain effects contained in the main (SO coupling-free) Hamiltonian. Correspondingly, deformation potentials were obtained in the absence of SO coupling. We present results at the $\Gamma$ point only. All the values are given with respect to the average electrostatic (Hartree) potential of the unit cells in Table III.

\section{SIXTEEN-BAND HAMILTONIAN FOR WURTZITE CRYSTALS}

In order to provide an accurate description of the two energetically close conduction bands closest to the band gap in WZ GaAs within the perturbative approach of the $\mathbf{k} \cdot \mathbf{p}$ formalism beyond the eight-band model, remote conduction bands gain importance due to their coupling to the $\Gamma_{8}$ conduction band. We have thus employed a sixteen-band model taking the top three $p$-like valence bands, the $\Gamma_{7}$ and $\Gamma_{8}$ conduction bands, and the following three $p$-like conduction bands into account, each with their respective $|\uparrow\rangle$ and $|\downarrow\rangle$ component to a total of sixteen bands. The details of the Hamiltonian can be found together with a schematic plot of bands and coupling terms in Appendix B. In total, 24 parameters need to be determined by fitting to the $a b$ initio band structure, namely: $m_{\|}^{\mathrm{c}}, m_{\perp}^{\mathrm{c}}, m_{\|}, m_{\perp}(4)$, the Luttinger-like parameters $A_{i}$ and $A_{i}^{\mathrm{c}}$ with $i=1 . .6(12)$, and the optical matrix elements $P_{1}, P_{2}, \mathrm{P}_{1}^{\mathrm{c}}, \mathrm{P}_{2}^{\mathrm{c}}, \mathrm{P}_{1}^{\prime}, \mathrm{P}_{2}^{\prime}, \mathrm{P}_{1}^{\prime \prime \prime}$, and $\mathrm{P}_{2}^{\prime \prime \prime}(8)$.

\section{MULTIBAND PARAMETER EXTRACTION}

The parameter sets required in $\mathbf{k} \cdot \mathbf{p}$ models of arbitrary complexity can be obtained by fitting the respective $\mathbf{k} \cdot \mathbf{p}$ band structure to an $a b$ initio band structure as, e.g., the one obtained in Sec. II. While some of these parameters can be read directly from the band structure at $\Gamma$ (e.g., the band gap, CF and SO splitting parameters), effective masses and Luttinger-like parameters as well as optical matrix elements can be determined directly from nonlocal empirical pseudopotentials or $a b$ initio models with some limitations [26-28] but are best determined by numerical fitting. This can be done using gradient minimization schemes readily available in standard mathematics libraries for $\mathbf{k} \cdot \mathbf{p}$ models with a limited level of sophistication as, e.g., six- or eight-band models. However, such schemes commonly fail for more complex multiband models due to a large number of local minima in the difference between original and fitted band structure spanned by the amount of parameters to be fitted. To avoid trapping in local minima, it is thus necessary to sample the whole, multidimensional search space. For the example of an eight-band model for ZB crystals, band gap, and SO splitting can be obtained directly from the band structure, whereas the electron effective mass $m_{\mathrm{e}}$, three Luttinger parameters $\gamma_{1,2,3}$, and the Kane parameter $E_{\mathrm{P}}$ need to be determined by fitting. With $n_{\mathbf{k}}$ data points of the $a b$ initio input band structure and

TABLE III. Deformation potentials (in the absence of SO coupling) for WZ GaAs given as the partial derivative of the bands energies at the $\Gamma$ point. All the energies are given with respect to the average Hartree potential of the unit cell. Units are $\mathrm{eV}$.

\begin{tabular}{lccccc}
\hline \hline Band ordering $(\epsilon=0)$ & Band character & $\frac{\partial\left(E_{i}-E_{\mathrm{H}}^{\mathrm{av}}\right)}{\partial \epsilon_{1}}$ & $\frac{\partial\left(E_{i}-E_{\mathrm{H}}^{\mathrm{av}}\right)}{\partial \epsilon_{3}}$ & $\frac{\partial\left(E_{i}-E_{\mathrm{H}}^{\mathrm{av}}\right)}{\partial \epsilon_{4}}$ & 0 \\
\hline $\mathrm{VBE}-1$ & $z$-like & -6.39 & -13.88 & 0 & 0.15 \\
$\mathrm{VBE}$ & $y$-like & -6.79 & -7.24 & $-3.47([x y]-$ like $)$ \\
$\mathrm{VBE}$ & $x$-like & -13.82 & -7.24 & -0.15 & $3.47([x-y]-$ like $)$ \\
$\mathrm{CBE}$ & $s$-like & -16.91 & -20.30 & 0 & 0 \\
$\mathrm{CBE}+1$ & $s$-like & -17.98 & -0.62 & 0.20 & $-3.47([x y]-$ like $)$ \\
$\mathrm{CBE}+2$ & $x$-like & -15.11 & -4.65 & -0.20 & $3.47([x-y]$-like $)$ \\
$\mathrm{CBE}+2$ & $y$-like & -8.22 & -4.65 & 0 & 0 \\
$\mathrm{CBE}+4$ & $z$-like & -6.32 & -17.41 & & 0 \\
\hline \hline
\end{tabular}




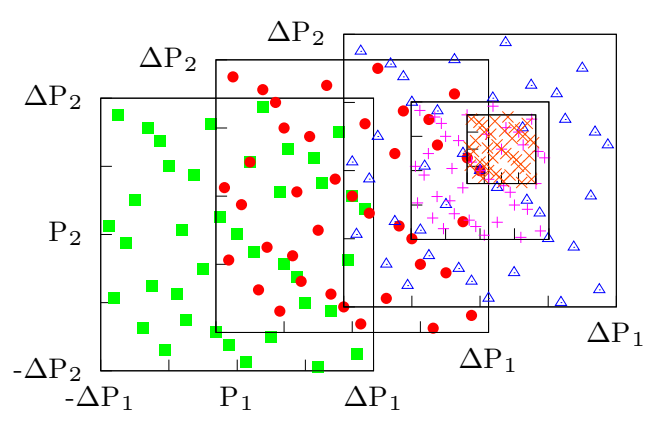

FIG. 2. Schematic of the low-discrepancy parameter fitting algorithm. First, the search space is moved with identical ranges until the minimum remains in its center (green squares, red bullets, blue triangles). Once this is achieved, the search space is reduced to increase the accuracy of the parameters (magenta " + " and orange "x").

assuming a discretization of $N$ points in each dimension of the parameter set $P$, we minimize the absolute difference between input band structure $\epsilon$ and the one obtained from the fit:

$$
v=\sum_{n_{\mathbf{k}}} \operatorname{eig}\left[\hat{H}_{\mathbf{k p}}\left(P_{i}\right)-\epsilon\right]_{i=1 . . N} \longrightarrow \mathrm{MIN} !
$$

Let us assume that we have a band structure of $n_{\mathbf{k}}=$ 150 sample points and that a single eigenvalue problem can be solved within $50 \mu$ s of single-processor time. For an eight-band model for zinc-blende crystals and taking $N=10$ discretization points in each direction of the five-dimensional parameter set $P$, this results in a computational effort of about $12 \mathrm{~min}$. The computational effort increases exponentially in the numbers of parameters to be fitted. For example, for the WZ structure the dimension of the parameter space doubles and the computational effort would already exceed two years of single processor time. Of course, this minimization problem is perfectly suited for parallelization, but the computational effort nevertheless remains extreme and more sophisticated models than the eight-band formalism, such as full-zone models for indirect band gap semiconductors that require even larger parameter sets, are completely beyond the reach of today's computational capabilities.

This curse of dimensionality can be overcome by stochastic optimization based on randomly chosen search points for the global minimum. Low-discrepancy points are a natural substitute for random numbers. Such quasi-Monte Carlo methods provide a higher accuracy with fewer evaluations of the objective function in Eq. (1) [29]. Many practical studies have shown that Sobol points [30,31] are superior to many other choices of low-discrepancy point sets. For this reason, we search the whole $N_{\mathrm{P}}$-dimensional parameter set $P$ by using properly scaled points from an $N_{\mathrm{P}}$-dimensional Sobol sequence. In this manner, we can keep the number of search points fixed along different dimensionalities of the parameter space, still being able to obtain values close to the global minimum. The respective fitting scheme is illustrated in Fig. 2 for a two-dimensional parameter space. In a first step, the parameter space sampled by the Sobol sequence is spanned by an initial estimate of each parameter together with a respective search range. Some parameters may be known with reasonable accuracy from literature or experience and will have a small surrounding search range whereas this range will be larger for those parameters of which no reference is known. The $N_{\mathrm{P}}$-dimensional Sobol sequence is then mapped on the search ranges of all parameters and the best fit is obtained within all parameter sets defined in this manner (green squares in Fig. 2). Without reducing the size of the search space around all parameters, a new sequence of parameter sets is then spanned around the best suited set obtained before, such that the whole search space moves towards the best set (green squares and blue triangles). Once the best suited parameter set is found and remains unchanged when setting up a new sequence of parameter sets, the search space is reduced by a factor two in each parameter search range such that the numerical accuracy of all parameters increases either to a defined number of search space reductions or until any other chosen convergence criterion is fulfilled (magenta plus and orange crosses).

The objective function in Eq. (1) can furthermore be modified such that priorities of selected $\mathbf{k}$ points as well as those of particular bands that are of pronounced importance, can be increased via individual weights. In order to ensure a good fit at specific high-symmetry points, one can thus easily increase the importance of these points by assigning higher weights to $\mathbf{k}$ values in their vicinity. Finally, we can evaluate parameter sets within the search space with respect to the degree of nonellipticity of a Hamiltonian operator employing them.

We demonstrate our fitting scheme for the example of a sixteen-band $\mathbf{k} \cdot \mathbf{p}$ model that takes the top three valence and bottom five conduction bands into account, each with their respective spin-up and spin-down components. We have extracted these bands from the ab initio band structure computed above in order to determine the $\mathbf{k} \cdot \mathbf{p}$ parameters for the model as outlined in Appendix B. This particular model requires 24 parameters that are to be fitted to the $a b$ initio band structure. The $\mathbf{k} \cdot \mathbf{p}$ Hamiltonian is constructed in a basis consisting of $\mathrm{P}$ - and S-like bands. Correspondingly, we first evaluate the orbital character of the ab initio bands involved.

Figure 3 shows the character of the $a b$ initio bands to which the $\mathbf{k} \cdot \mathbf{p}$ band structure will be fitted. The valence bands $\Gamma_{7 \mathrm{v}+}, \Gamma_{7 \mathrm{v}-}$, and $\Gamma_{9 \mathrm{v}}$ exhibit predominantly a P-like character (red solid, dashed, and dash-dotted lines), $\Gamma_{7 \mathrm{v}+}$ furthermore contains visible S-like contributions (black solid lines). The two conduction bands closest to the band gap, $\Gamma_{7 \mathrm{c}}$ and $\Gamma_{8 c}$, are mostly of $\mathrm{S}$ character but also with visible P-like contributions. The following conduction band states are predominantly of $\mathrm{P}$ character with S-like contributions in $\Gamma_{9 c}$ and $\Gamma_{7 c+}$ around the high-symmetry A point. Moreover, nonzero D-like character is seen in these conduction bands (green solid lines), however the impact of D-like bands remains negligible. The character analysis thus reveals that $\mathrm{S}$ - and P-like bands in fact represent a reasonable basis for the sixteen band model.

Correspondingly, Fig. 4 shows the sixteen-band $\mathbf{k} \cdot \mathbf{p}$ band structure for WZ GaAs crystals that was fitted to the above $a b$ initio band structure. Priority was given to the bands closest to the band gap, namely the bottom two conduction bands and the top two valence bands as they dominate the electronic structure of WZ GaAs. The $\mathbf{k} \cdot \mathbf{p}$ band structure is in good agreement with the $a b$ initio one throughout wide parts of the $\mathrm{BZ}$ for the bands of pronounced priority. This is particularly 

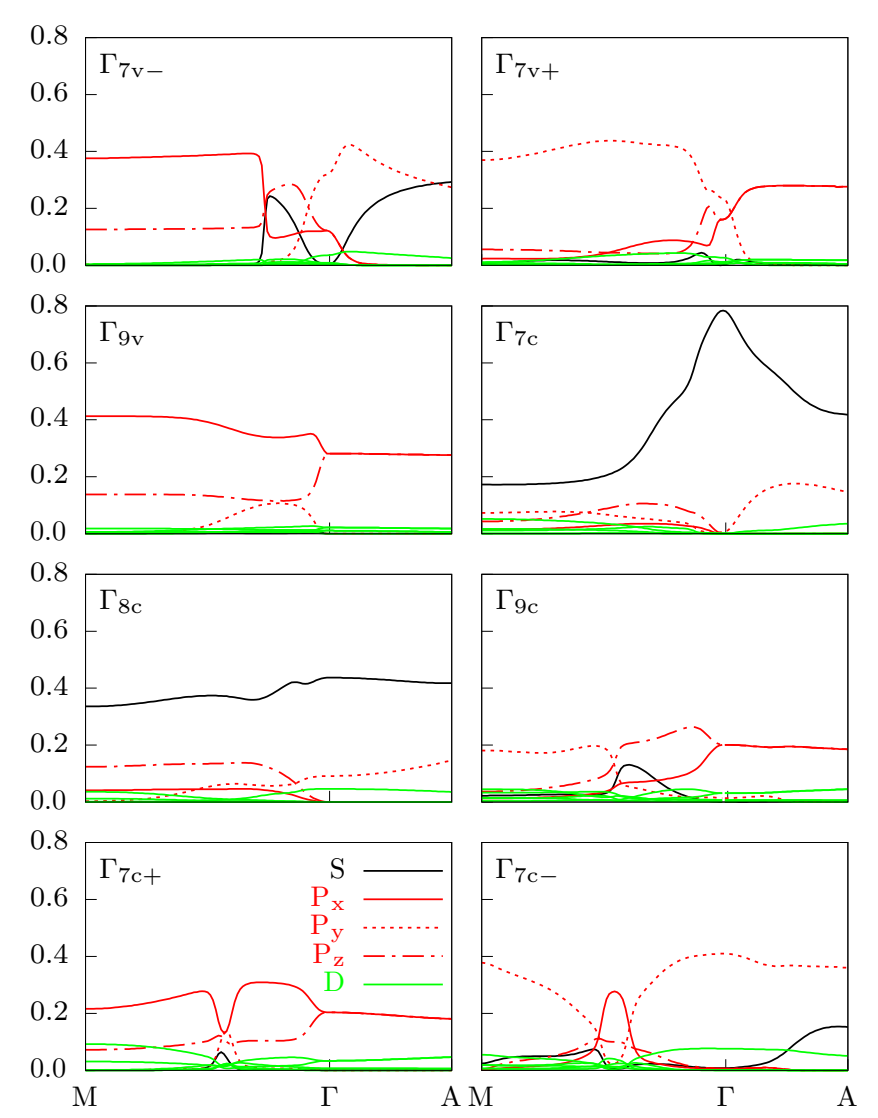

FIG. 3. Character of S (black), P (red), and D orbitals (green) of the top six valence and bottom ten conduction bands of the $a b$ initio band structure that is used for the fitting procedure. As all bands are pairwise almost degenerate, we evaluate here only one of each pair of bands for the sake of readability. Note that the sum of all contributions to a band is not necessarily one as the underlying atomcentered spherical harmonics do not represent a complete basis.

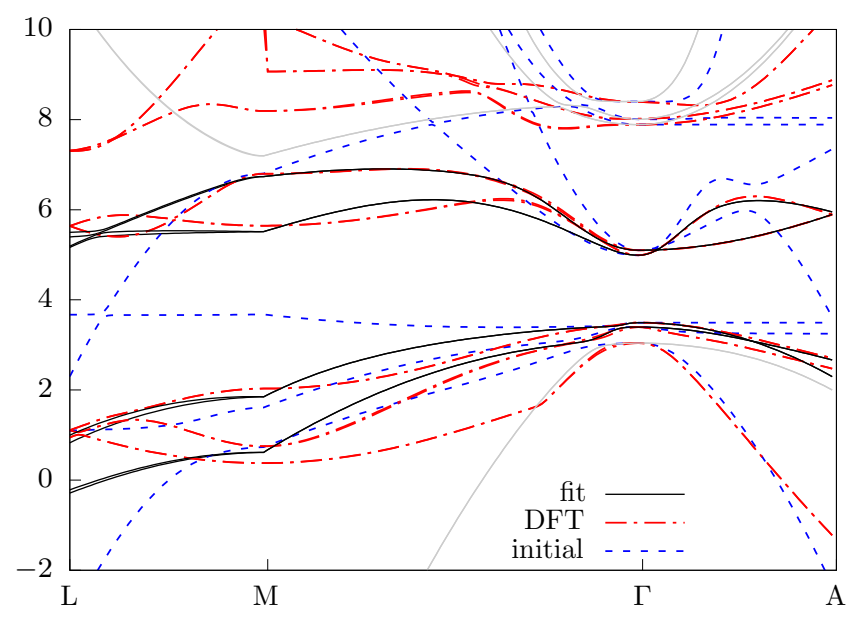

FIG. 4. Fit (black and gray solid) of a sixteen-band WZ GaAs $\mathbf{k} \cdot \mathbf{p}$ band structure to the above $a b$ initio band structure (red dashdotted). The blue dashed band structure was obtained from the initial guess parameter set around which the initial Sobol-sequence generated parameter sets were constructed. Priority was given to the four valence and conduction bands closest to the band gap (black solid).
TABLE IV. Fitting parameters and band splittings obtained for WZ GaAs within the 16-band $\mathbf{k} \cdot \mathbf{p}$ model. The band splitting parameters $\Delta_{1,2,3}^{(c)}$ can be extracted directly from the eigenenergies at $\Gamma$. The splittings $\Delta_{1,2,3}^{c v}$ were found to be negligibly small and are therefore not listed. Values are given without $(\varepsilon=0.0)$ and with ellipticity conditions taken into account $(\varepsilon=0.2)$.

\begin{tabular}{lrrcrr}
\hline \hline Parameter & $\varepsilon=0.0$ & $\varepsilon=0.2$ & Parameter & $\varepsilon=0.0$ & $\varepsilon=0.2$ \\
\hline $\mathrm{m}_{\|}^{e}\left(\mathrm{~m}_{0}\right)$ & 0.032 & 0.048 & $\mathrm{~m}_{\|}^{c}\left(\mathrm{~m}_{0}\right)$ & 1.117 & 1.443 \\
$\mathrm{~m}_{\perp}^{e}\left(\mathrm{~m}_{0}\right)$ & 0.090 & 0.075 & $\mathrm{~m}_{\perp}^{c}\left(\mathrm{~m}_{0}\right)$ & -0.352 & -0.648 \\
$\mathrm{~A}_{1}\left(\hbar^{2} / \mathrm{m}_{0}\right)$ & -1.386 & -1.484 & $\mathrm{~A}_{1}^{c}\left(\hbar^{2} / \mathrm{m}_{0}\right)$ & -0.951 & 0.354 \\
$\mathrm{~A}_{2}\left(\hbar^{2} / \mathrm{m}_{0}\right)$ & -0.816 & -0.870 & $\mathrm{~A}_{2}^{c}\left(\hbar^{2} / \mathrm{m}_{0}\right)$ & -0.375 & -0.799 \\
$\mathrm{~A}_{3}\left(\hbar^{2} / \mathrm{m}_{0}\right)$ & 0.003 & 0.508 & $\mathrm{~A}_{3}^{c}\left(\hbar^{2} / \mathrm{m}_{0}\right)$ & 4.772 & 10.110 \\
$\mathrm{~A}_{4}\left(\hbar^{2} / \mathrm{m}_{0}\right)$ & 0.159 & 0.553 & $\mathrm{~A}_{4}^{c}\left(\hbar^{2} / \mathrm{m}_{0}\right)$ & 2.687 & 1.326 \\
$\mathrm{~A}_{5}\left(\hbar^{2} / \mathrm{m}_{0}\right)$ & -1.241 & -0.296 & $\mathrm{~A}_{5}^{c}\left(\hbar^{2} / \mathrm{m}_{0}\right)$ & -4.896 & -1.084 \\
$\mathrm{~A}_{6}\left(\hbar^{2} / \mathrm{m}_{0}\right)$ & 0.176 & -0.115 & $\mathrm{~A}_{6}^{c}\left(\hbar^{2} / \mathrm{m}_{0}\right)$ & -0.549 & -0.148 \\
$\mathrm{P}_{1}(\mathrm{eV})$ & 1.369 & 1.844 & $\mathrm{P}_{1}^{c}(\mathrm{eV} \AA)$ & 0.062 & 0.369 \\
$\mathrm{P}_{2}(\mathrm{eV} \AA)$ & 2.409 & 1.316 & $\mathrm{P}_{2}^{c}(\mathrm{eV} \AA)$ & 1.342 & 1.174 \\
$\mathrm{P}_{1}^{\prime}(\mathrm{eV} \AA)$ & 1.494 & -1.859 & $\mathrm{P}_{1}^{\prime \prime \prime}(\mathrm{eV} \AA)$ & 13.939 & 12.967 \\
$\mathrm{P}_{2}^{\prime}(\mathrm{eV} \AA)$ & 8.033 & 5.697 & $\mathrm{P}_{2}^{\prime \prime \prime}(\mathrm{eV} \AA)$ & 6.132 & 7.271 \\
$\Delta_{1}(\mathrm{eV})$ & 0.180 & 0.180 & $\Delta_{1}^{c}(\mathrm{eV})$ & -0.409 & -0.409 \\
$\Delta_{2,3}(\mathrm{eV})$ & 0.124 & 0.124 & $\Delta_{2,3}^{c}(\mathrm{eV})$ & -0.074 & -0.074 \\
\hline \hline
\end{tabular}

remarkable as the $\mathbf{k} \cdot \mathbf{p}$ perturbation theory commonly allows an accurate description of a band structure only in the close vicinity of high-symmetry points and is not expected to keep this accuracy throughout the BZ. In comparison, the band structure obtained from the initial parameters is depicted in Fig. 4 in blue dashed lines and exhibits very poor agreement with the ab initio band structure. It is therefore not necessary to start the minimization with initial parameters that are already close to the final ones, however, the smaller the search range for each parameter, the faster the minimization will deliver a good fit. To quantify the quality of the fit, we compute the improvement of $v$ with respect to the initial guess, $\mathrm{I}=1-v / v_{\text {init }}$, as absolute values of $v$ are unhelpful, given their dependence on a number of factors such as the number of $\mathbf{k}$ values or band and $\mathbf{k}$ prioritization. For the current fit, we obtain $\mathrm{I}=89.7 \%$.

The band structure parameters obtained from the fit are presented in Tab. IV and could, in principle, serve as input for electronic-structure simulations of WZ GaAs heterostructures using the more sophisticated sixteen-band $\mathbf{k} \cdot \mathbf{p}$ model. Details of the initial parameters, search spaces, and priorities can be found in Appendix C.

However, from the curvature of the bands around the BZ boundaries, it can already be expected that spurious solutions will arise if these parameters are employed in a single-particle calculation of a heterostructure. For example, the conduction band bending downwards at the $\mathrm{L}$ point will continue deep into the band gap, resulting in energy minima that are a consequence of the incorrect band structure.

This can also be seen in Fig. 5 where the density of states (DOS) reproduced from the sixteen-band $\mathbf{k} \cdot \mathbf{p}$ band structure above is shown, obtained from sampling throughout the whole BZ. The band gap is indicated with two black solid lines. It can be seen that non-negligible band contributions exist inside the band gap $\left(\mathrm{p}_{\mathrm{g}}=2.6 \%\right.$ of all eigenvalues obtained by sampling throughout the first BZ), which is an indicator for 


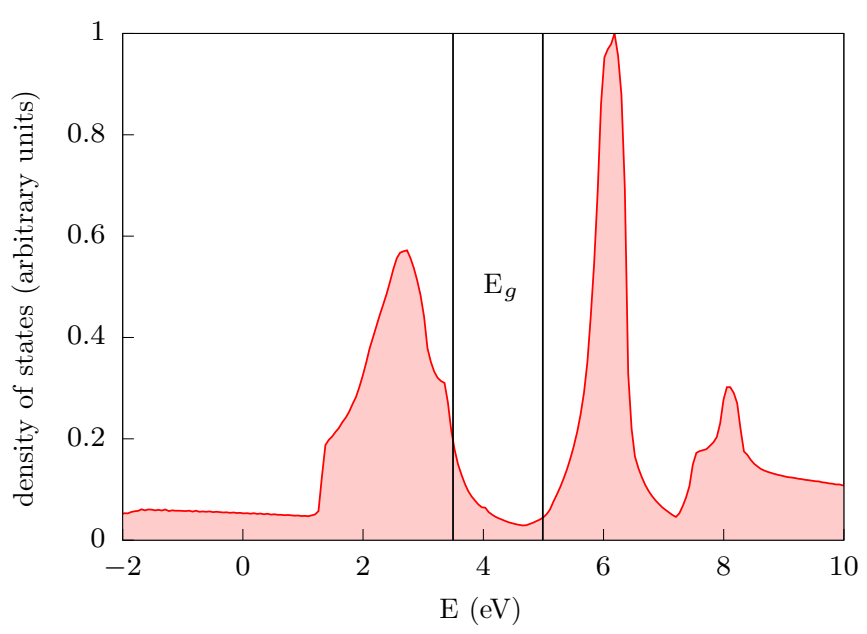

FIG. 5. Density of states of the $\mathbf{k} \cdot \mathbf{p}$ band structure of the sixteen-band model shown in Fig. 4.

the above unphysical band bending somewhere inside the BZ that can potentially induce spurious solutions when a 16-band model with the corresponding material parameters obtained from our fit is applied to study the electronic properties of a nanostructure.

\section{ELLIPTICITY CONSIDERATIONS}

In the following, we include ellipticity conditions in our fitting scheme in order to reduce the unphysical DOS inside the band gap. The parameters $\varrho_{\mathrm{c}}$ and $\varrho_{\mathrm{v}}$ as defined in Eq. (A20) in Appendix D quantify the degree of nonellipticity and are thus a measure for the quality of the parameter set with respect to robustness against spurious solutions (for more details see Appendix D), where small values of $\varrho_{\mathrm{c}}$ and $\varrho_{\mathrm{v}}$ represent robust parameter sets. The sum of both, $\varrho=\varrho_{\mathrm{c}}+\varrho_{\mathrm{v}}$, is then multiplied with the cost function $v$ from the initial guess and the parameter $\varepsilon$ that defines the priority to be given to ellipticity. The respective value is then added to the cost function $v$ for each evaluated parameter set. Figure 6 shows the impact of imposing ellipticity conditions on our band structure fit for priority values of $\varepsilon=0.1,0.2$, and 0.3 . It can be seen that an increased priority $\varepsilon$ of the ellipticity conditions in fact reduces the DOS inside the band gap, however, at the cost of a reduced quality of the band structure fit, in particular in the remote areas of the BZ (see values for I provided in the plot). The lowest percentage of eigenvalues inside the band gap obtained from sampling throughout the whole $\mathrm{BZ}$ is obtained for $\varepsilon=$ 0.2 , with $\mathrm{p}_{\mathrm{g}}=1.2 \times 10^{-5}$. Correspondingly, this parameter set is most suited to be applied in heterostructure modeling to avoid the occurrence of spurious solutions. The respective parameters are given in Table IV. For $\varepsilon=0.3$, the percentage of eigenvalues inside the band gap increases again, which can be attributed to the fact that coupling linear in $\mathbf{k}$ is neglected in the ellipticity considerations [cf. Eqs. (A17) and (A19) in Appendix D]. This can also be observed when comparing the respective band structures for $\varepsilon=0.2$ (red dash-dotted) and 0.3 (blue dashed) where one of the conduction bands of the latter one is clearly seen to drop into the band gap.

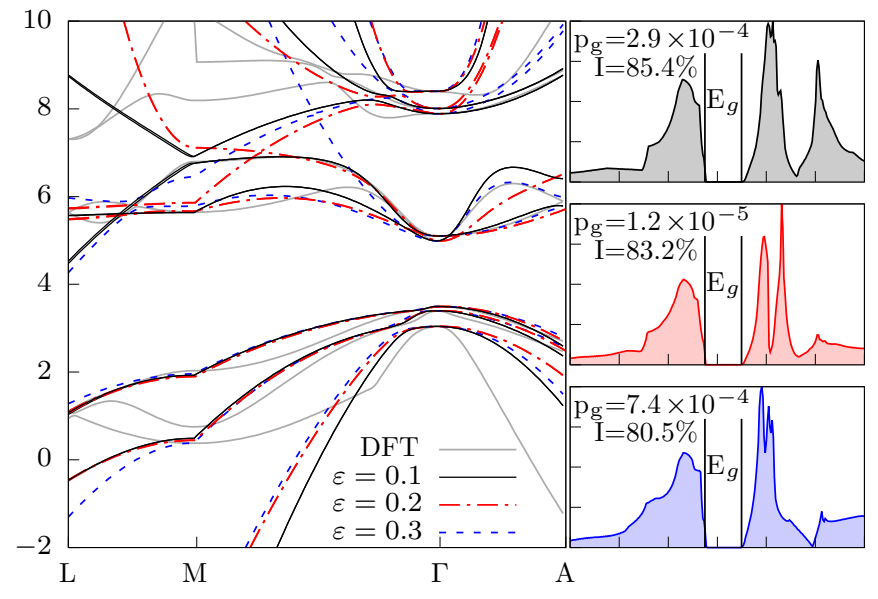

FIG. 6. Left: band structures obtained from fitting for $\varepsilon=0.1$ (black solid), 0.2 (red dash-dotted), and 0.3 (blue dashed line). The DFT target band structure is shown in gray. Right: the respective density of states for $\varepsilon=0.1,0.2,0.3$ (top, middle, bottom). The percentage of the band structure inside the band gap, $\mathrm{p}_{\mathrm{g}}$, as well as the improvement towards the initial guess band structure, I, are provided with each plot.

If we compare the parameters resulting from the fit (cf. Table IV) with literature values where available [32-34], we see some significant deviations from existing parameters. In particular, our electron effective masses are quite different from established values, a fact that can also be seen when looking at the band structure in the vicinity of $\Gamma$. However, this is not surprising at this point as the intention of our fitting, and thus the selection of priorities for specific bands and high-symmetry points was to achieve a good fit throughout the whole BZ rather than a good fit of the effective masses in the close vicinity of the zone center $\Gamma$, where electronic-structure parameters are commonly extracted.

We emphasize that for simulations of larger heterostructures or electron transport simulations in devices, it is reasonable to extract electronic band structure parameters from the closer vicinity of $\Gamma$ only, which here is the dominant region of the BZ. Within a full-zone fit, the agreement between the $\mathbf{k} \cdot \mathbf{p}$ and the $a b$ initio band structure at $\Gamma$ naturally suffers from the compromise of finding a reasonably good fit not only around $\Gamma$ but throughout the whole BZ.

\section{PARAMETERS FOR A TEN-BAND MODEL}

As the application of a sixteen-band model for the calculation of the electronic properties of a heterostructure represents a much larger computational effort than the well-established eight-band model, we have furthermore extracted a parameter set for a ten-band model required for WZ GaAs and given in detail in Ref. [12] including the top three valence and the bottom two conduction bands in the closer vicinity of the center of the BZ, $\Gamma$. For this model, a total of 13 unknown parameters was determined. The corresponding parameter set is shown in Table V. Of course, the description of the band structure is accurate only in the center of the BZ (cf. Fig. 7) due to the limited basis of the ten-band model. Here, we have also incorporated the ellipticity conditions outlined in 
TABLE V. Fitting parameters obtained for WZ GaAs within the ten-band $\mathbf{k} \cdot \mathbf{p}$ model. The parameter $\mathrm{P}_{2}^{\mathrm{cv}}$ couples the $\Gamma_{8}$ conduction band to the top $\Gamma_{9 v}$ valence band within the ten-band model employed (the Hamiltonian is given in Ref. [12], $\mathrm{P}_{2}^{\mathrm{cv}}$ here enters via: $\left.R=i\left(\partial_{x}+i \partial y\right) \mathrm{P}_{2}^{\mathrm{cv}}\right)$.

\begin{tabular}{lllr}
\hline \hline $\mathrm{m}_{\|}^{e}\left(\mathrm{~m}_{0}\right)$ & 0.0582 & $\mathrm{~A}_{1}\left(\hbar^{2} / \mathrm{m}_{0}\right)$ & -13.666 \\
\hline $\mathrm{m}_{\perp}^{e}\left(\mathrm{~m}_{0}\right)$ & 0.0979 & $\mathrm{~A}_{2}\left(\hbar^{2} / \mathrm{m}_{0}\right)$ & -7.088 \\
$\mathrm{~m}_{\|}^{c}\left(\mathrm{~m}_{0}\right)$ & 2.549 & $\mathrm{~A}_{3}\left(\hbar^{2} / \mathrm{m}_{0}\right)$ & 6.972 \\
$\mathrm{~m}_{\perp}^{c}\left(\mathrm{~m}_{0}\right)$ & 0.1276 & $\mathrm{~A}_{4}\left(\hbar^{2} / \mathrm{m}_{0}\right)$ & 2.727 \\
$\mathrm{P}_{1}(\mathrm{eV} \AA)$ & 2.270 & $\mathrm{~A}_{5}\left(\hbar^{2} / \mathrm{m}_{0}\right)$ & 4.952 \\
$\mathrm{P}_{2}(\mathrm{eV} \AA)$ & 2.996 & $\mathrm{~A}_{6}\left(\hbar^{2} / \mathrm{m}_{0}\right)$ & 2.641 \\
$\mathrm{P}_{2}^{\text {cv }}(\mathrm{eV} \AA)$ & 1.301 & & \\
\hline \hline
\end{tabular}

Ref. [15] to the valence band part of the Hamiltonian [cf. Eq. (A17) in Appendix D] to reduce the risk of spurious solutions if our parameter set is applied to a heterostructure. Ellipticity was here given a priority of $\varepsilon=0.5$.

We note that our scheme still requires some knowledge on realistic search ranges for each parameter and a fitted parameter set will only be as good as the initial search range chosen for it. In particular, for large search spaces with small initial Sobol sequences, the algorithm can get trapped into local minima and will not see other, global ones. It is thus necessary to carefully evaluate the convergence of the band structure with the number of parameter sets generated using the Sobol sequence. Some of the parameters, e.g., effective masses, can be estimated from the curvature at the $\Gamma$ point, but for others a respectively large search space is required. Moreover, band- and k-point priorities can be adjusted to achieve a good fit only for some of the bands employed, leaving both a certain degree of arbitrariness in the fitting process and large deviations from the $a b$ initio band structure for bands of low priority (cf. gray bands in Fig. 4).

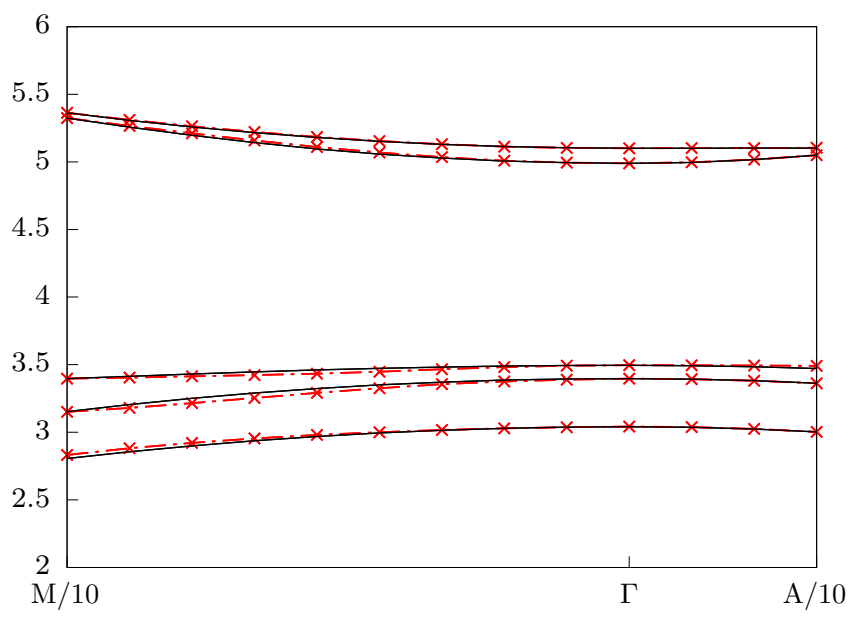

FIG. 7. Ab initio band structure (red dash-dotted lines, ' $x$ ' symbols) and fit using a ten-band $\mathbf{k} \cdot \mathbf{p}$ model (black solid) in the vicinity of $\Gamma$. The respective parameters are listed in Table $\mathrm{V}$.

\section{SUMMARY}

We have presented a numerically efficient fitting scheme to obtain $\mathbf{k} \cdot \mathbf{p}$ electronic structure parameters for multiband $\mathbf{k} \cdot \mathbf{p}$ models beyond the well-established eight-band approach from up-to-date $a b$ initio band structures. Our scheme employs low-discrepancy sequences to sample multidimensional parameter spaces for more sophisticated $\mathbf{k} \cdot \mathbf{p}$ models. In addition, it allows direct control of priorities of specific $\mathbf{k}$ points and bands that are of pronounced importance for the electronic structure to ensure a highly accurate fit within selected regions. Furthermore, the likelihood to encounter spurious solutions can be reduced by introducing nonellipticity conditions during the fitting process. We have presented material parameters for both a 16-band $\mathbf{k} \cdot \mathbf{p}$ model that was developed within this work as well as a ten-band model which is better suited for computationally inexpensive simulations of the electronic structure of heterostructures containing GaAs in the WZ phase. While the focus of the present study was on the material parameters of GaAs, we note that the influence of additional conduction bands plays a significant role for other III-V WZ semiconductors: The energetical difference between the lowest conduction bands reported for $\mathrm{GaSb}$ and InP are 87 and $353 \mathrm{meV}$, respectively, and the order of the bottom two conduction bands in WZ AlP, AlAs, AlSb, and $\mathrm{GaP}$ is even reversed [34]. Finally, we remark that our scheme is not limited to semiconductors but can provide parameters for all band structures that can be computed from a Hamiltonian.

\section{ACKNOWLEDGMENTS}

Computational resources for this project were provided by CSC-IT Center for Science, Finland, through Project No. 2000634. M.A.C. acknowledges Academy of Finland support through Grant No. 310574. O.M. acknowledges support from the Deutsche Forschungsgemeinschaft (DFG, German Research Foundation) under Germany's Excellence Strategy - EXC2046: MATH+ Berlin Mathematics Research Center (project AA2-5).

\section{APPENDIX}

\section{Density functional theory}

In view of the scarcity of data available on WZ GaAs, to accurately model its electronic structure we fitted the screening parameter $\mu$ of the HSE hybrid functional [35] to yield the experimental zero-temperature band gap for ZB GaAs first, which is well established at $1.519 \mathrm{eV}$. This is achieved in our calculations, in the absence of SO coupling, for $\mu=0.138 \AA^{-1}$. However, SO coupling reduces the size of the band gap, which means a lower value of $\mu$ is required to reproduce the experimental gap with the SO interaction enabled. Therefore we chose $\mu=0.089 \AA^{-1}$, which yields a good agreement with the experimental value. Compared to the $\mu=0.3 \AA^{-1}$ and $\mu=0.2 \AA^{-1}$ screening parameters of HSE03 and HSE06, our customized functional is less screened. We explored a combined optimization of screening parameter and mixing parameter $\alpha$, to try and improve the simultaneous description of both band gap and 
lattice parameter. However, only marginal improvement was achieved for reasonable values of $\alpha$. Perfect agreement for both quantities without SO coupling was obtained for the tuple $(\mu, \alpha)=\left(0.8 \AA^{-1}, 1.45\right)$. In view of the difficulty to optimize both parameters and the fact that the choice of $\alpha=$ 0.25 is theoretically justified [36], we decided to settle for $(\mu, \alpha)=\left(0.089 \AA^{-1}, 0.25\right)$. The DFT code used was VASP [37], which is based on projector-augmented wave (PAW) potentials [38,39] and plane-wave basis sets. We chose a cutoff energy for the plane waves of $350 \mathrm{eV}$. The grids used for BZ integration were chosen differently for structural and elastic properties (finer mesh) and electronic structure (coarser mesh). For a GaAs ZB primitive unit cell (two atoms) we found that a $10 \times 10 \times 10$ mesh was enough to converge the total energy below $0.1 \mathrm{meV}$. For the electronic structure calculations, we used a $6 \times 6 \times 6$ mesh. The motivation for using a finer $k$ mesh for structural properties is twofold. First, band structures are less sensitive to the density of $k$ points used. Second, for SO calculations, the SO coupling breaks the symmetry of the BZ, leading to a significantly larger number of nonequivalent $k$ points; using dense grids becomes prohibitively expensive. For the evaluation of the Coulomb kernel (for the exact-exchange calculation) we used a $k$-point reduction scheme whereby the number of $k$ points in the grid was halved along each reciprocal lattice direction. This is known as "downsampling" [40]. All the other simulations were carried out maintaining a density of $k$ points as close as possible to those specified above. The semicore $d$ electrons of $\mathrm{Ga}$ were included explicitly in the calculation as valence electrons.

\section{Sixteen-band Hamiltonian}

We start with the assumption that the relevant near-band gap states are spanned by the $p$-like valence bands $X_{\mathrm{v}}, Y_{\mathrm{v}}, Z_{\mathrm{v}}$ followed by the $s$-like conduction band $S$ (commonly the conduction band closest to the band gap), three $p$-like conduction bands $X_{\mathrm{c}}, Y_{\mathrm{c}}$, and $Z_{\mathrm{c}}$ and another $s$-like band $S^{\prime}$. We follow the procedure used in Ref. [24] with the basis set:

$|S \uparrow\rangle,\left|X_{\mathrm{v}} \uparrow\right\rangle,\left|Y_{\mathrm{v}} \uparrow\right\rangle,\left|Z_{\mathrm{v}} \uparrow\right\rangle,|S \downarrow\rangle,\left|X_{\mathrm{v}} \downarrow\right\rangle,\left|Y_{\mathrm{v}} \downarrow\right\rangle,\left|Z_{\mathrm{v}} \downarrow\right\rangle$ for the top $p$-like valence bands and the (commonly bottom) $s$-like conduction band and $\left|S^{\prime} \uparrow\right\rangle,\left|X_{\mathrm{c}} \uparrow\right\rangle,\left|Y_{\mathrm{c}} \uparrow\right\rangle,\left|Z_{\mathrm{c}} \uparrow\right\rangle,\left|S^{\prime} \downarrow\right\rangle,\left|X_{\mathrm{c}} \downarrow\right\rangle,\left|Y_{\mathrm{c}} \downarrow\right\rangle,\left|Z_{\mathrm{c}} \downarrow\right\rangle$ for the respective following conduction bands. The Hamiltonian then reads:

$$
\hat{H}=\left(\begin{array}{cc}
G_{1}^{\mathrm{c}}(\mathbf{k})+G_{2}^{\mathrm{c}}(\mathbf{k}) & \Gamma \\
-\Gamma^{\dagger} & G_{1}(\mathbf{k})+G_{2}(\mathbf{k})
\end{array}\right)
$$

with:

$$
\begin{aligned}
& G_{1}^{\mathrm{c}}(\mathbf{k})=\left(\begin{array}{cccccccc}
E_{1}^{\mathrm{c}} & i P_{2}^{\mathrm{c}} k_{x} & i P_{2}^{\mathrm{c}} k_{y} & i P_{1}^{\mathrm{c}} k_{z} & 0 & 0 & 0 & 0 \\
-i P_{2}^{\mathrm{c}} k_{x} & E_{2}^{\mathrm{c}} & -i \Delta_{2}^{\mathrm{c}} & 0 & 0 & 0 & 0 & \Delta_{3}^{\mathrm{c}} \\
-i P_{2}^{\mathrm{c}} k_{y} & i \Delta_{2}^{\mathrm{c}} & E_{2}^{\mathrm{c}} & 0 & 0 & 0 & 0 & -i \Delta_{3}^{\mathrm{c}} \\
-i P_{1}^{\mathrm{c}} k_{z} & 0 & 0 & E_{2}^{\mathrm{c}}-\Delta_{\mathrm{CF}}^{\mathrm{c}} & 0 & -\Delta_{3}^{\mathrm{c}} & i \Delta_{3}^{\mathrm{c}} & 0 \\
0 & 0 & 0 & 0 & E_{1}^{\mathrm{c}} & i P_{2}^{\mathrm{c}} k_{x} & i P_{2}^{\mathrm{c}} k_{y} & i P_{1}^{\mathrm{c}} k_{z} \\
0 & 0 & 0 & -\Delta_{3}^{\mathrm{c}} & -i P_{2}^{\mathrm{c}} k_{x} & E_{2}^{\mathrm{c}} & i \Delta_{2}^{\mathrm{c}} & 0 \\
0 & 0 & 0 & -i \Delta_{3}^{\mathrm{c}} & -i P_{2}^{\mathrm{c}} k_{y} & -i \Delta_{2}^{\mathrm{c}} & E_{2}^{\mathrm{c}} & 0 \\
0 & \Delta_{3}^{\mathrm{c}} & i \Delta_{3}^{\mathrm{c}} & 0 & -i P_{1}^{\mathrm{c}} k_{z} & 0 & 0 & E_{2}^{\mathrm{c}}-\Delta_{\mathrm{CF}}^{\mathrm{c}}
\end{array}\right)
\end{aligned}
$$

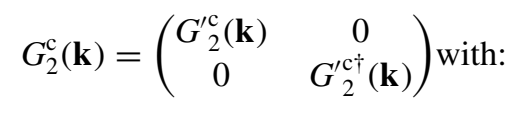

$$
\begin{aligned}
& {G^{\prime}}_{2}^{\mathrm{c}}(\mathbf{k})=\left(\begin{array}{cccc}
\tilde{A}_{2}^{\mathrm{c}}\left(k_{x}^{2}+k_{y}^{2}\right)+\tilde{A}_{1}^{\mathrm{c}} k_{z}^{2} & B_{x}^{\mathrm{c}} k_{y} k_{z} & B_{x}^{\mathrm{c}} k_{x} k_{z} & B_{z}^{\mathrm{c}} k_{x} k_{y} \\
B_{x}^{\mathrm{c}} k_{y} k_{z} & L_{1}^{\mathrm{c}} k_{x}^{2}+M_{1}^{\mathrm{c}} k_{y}^{2}+M_{2}^{\mathrm{c}} k_{z}^{2} & N_{1}^{\mathrm{c}} k_{x} k_{y} & N_{2}^{\mathrm{c}} k_{x} k_{z}-N_{3}^{\mathrm{c}} k_{x} \\
B_{x}^{\mathrm{c}} k_{x} k_{z} & N_{1}^{\mathrm{c}} k_{x} k_{y} & M_{1}^{\mathrm{c}} k_{x}^{2}+L_{1}^{\mathrm{c}} k_{y}^{2}+M_{2}^{\mathrm{c}} k_{z}^{2} & N_{2}^{\mathrm{c}} k_{y} k_{z}+N_{3}^{\mathrm{c}} k_{y} \\
B_{z}^{\mathrm{c}} k_{x} k_{y} & N_{2}^{\mathrm{c}} k_{x} k_{z}+N_{3}^{\mathrm{c}} k_{x} & N_{2}^{\mathrm{c}} k_{y} k_{z}-N_{3}^{\mathrm{c}} k_{y} & M_{3}^{\mathrm{c}}\left(k_{x}^{2}+k_{y}^{2}\right)+L_{2}^{\mathrm{c}} k_{z}^{2}
\end{array}\right) .
\end{aligned}
$$

The elements $\tilde{A}_{(1,2)}^{\mathrm{c}}, L_{(1,2)}^{\mathrm{c}}, M_{(1,2,3)}^{\mathrm{c}}$, and $N_{(1,2,3)}^{\mathrm{c}}$ are given by:

$$
\begin{gathered}
\tilde{A}_{1}^{\mathrm{c}}=\frac{1}{2 m_{\|}^{\mathrm{c}}}-\frac{\left(P_{2}^{\mathrm{c}}\right)^{2}}{E_{2}-E_{1}} \text { and } \tilde{A}_{2}^{\mathrm{c}}=\frac{1}{2 m_{\perp}^{\mathrm{c}}}-\frac{\left(P_{1}^{\mathrm{c}}\right)^{2}}{E_{2}-E_{1}} \\
L_{1}^{\mathrm{c}}=\frac{1}{2}\left(A_{2}^{\mathrm{c}}+A_{4}^{\mathrm{c}}+A_{5}^{\mathrm{c}}\right)-\frac{\left(P_{1}^{\mathrm{c}}\right)^{2}}{E_{2}-E_{1}} \text { and } L_{2}^{\mathrm{c}}=\frac{1}{2} A_{1}^{\mathrm{c}}+\frac{\left(P_{2}^{\mathrm{c}}\right)^{2}}{E_{2}-E_{1}} \\
M_{1}^{\mathrm{c}}=\frac{1}{2}\left(A_{2}^{\mathrm{c}}+A_{4}^{\mathrm{c}}-A_{5}^{\mathrm{c}}\right), \quad M_{2}^{\mathrm{c}}=\frac{1}{2}\left(A_{1}^{\mathrm{c}}+A_{3}^{\mathrm{c}}\right), \text { and } M_{3}^{\mathrm{c}}=\frac{1}{2} A_{2}^{\mathrm{c}} \\
N_{1}^{\mathrm{c}}=\frac{1}{2} A_{5}^{\mathrm{c}}+\frac{\left(P_{2}^{\mathrm{c}}\right)^{2}}{E_{2}-E_{1}}, \quad N_{2}^{\mathrm{c}}=\frac{1}{\sqrt{2}} A_{6}^{\mathrm{c}}+\frac{P_{1}^{\mathrm{c}} P_{2}^{\mathrm{c}}}{E_{2}-E_{1}} \text { and } N_{3}^{\mathrm{c}}=i \sqrt{2} A_{7}^{\mathrm{c}}
\end{gathered}
$$


and similarly:

$$
\begin{gathered}
G_{1}(\mathbf{k})=\left(\begin{array}{cccccccc}
E_{\mathrm{g}} & i P_{2} k_{x} & i P_{2} k_{y} & i P_{1} k_{z} & 0 & 0 & 0 & 0 \\
-i P_{2} k_{x} & 0 & -i \Delta_{2} & 0 & 0 & 0 & 0 & \Delta_{3} \\
-i P_{2} k_{y} & i \Delta_{2} & 0 & 0 & 0 & 0 & 0 & -i \Delta_{3} \\
-i P_{1} k_{z} & 0 & 0 & -\Delta_{\mathrm{CF}} & 0 & -\Delta_{3} & i \Delta_{3} & 0 \\
0 & 0 & 0 & 0 & E_{\mathrm{g}} & i P_{2} k_{x} & i P_{2} k_{y} & i P_{1} k_{z} \\
0 & 0 & 0 & -\Delta_{3} & -i P_{2} k_{x} & 0 & i \Delta_{2} & 0 \\
0 & 0 & 0 & -i \Delta_{3} & -i P_{2} k_{y} & -i \Delta_{2} & 0 & 0 \\
0 & \Delta_{3} & i \Delta_{3} & 0 & -i P_{1} k_{z} & 0 & 0 & -\Delta_{\mathrm{CF}}
\end{array}\right) \\
G_{2}(\mathbf{k})=\left(\begin{array}{cccc}
G_{2}^{\prime}(\mathbf{k}) & 0 \\
0 & G_{2}^{\prime \dagger}(\mathbf{k})
\end{array}\right)
\end{gathered}
$$

with:

$$
G_{2}^{\prime}(\mathbf{k})=\left(\begin{array}{cccc}
\tilde{A}_{2}\left(k_{x}^{2}+k_{y}^{2}\right)+\tilde{A}_{1} k_{z}^{2} & B_{\mathrm{x}} k_{y} k_{z} & B_{\mathrm{x}} k_{x} k_{z} & B_{\mathrm{z}} k_{x} k_{y} \\
B_{\mathrm{x}} k_{y} k_{z} & L_{1} k_{x}^{2}+M_{1} k_{y}^{2}+M_{2} k_{z}^{2} & N_{1} k_{x} k_{y} & N_{2} k_{x} k_{z}-N_{3} k_{x} \\
B_{\mathrm{x}} k_{x} k_{z} & N_{1} k_{x} k_{y} & M_{1} k_{x}^{2}+L_{1} k_{y}^{2}+M_{2} k_{z}^{2} & N_{2} k_{y} k_{z}+N_{3} k_{y} \\
B_{\mathrm{z}} k_{x} k_{y} & N_{2} k_{x} k_{z}+N_{3} k_{x} & N_{2} k_{y} k_{z}-N_{3} k_{y} & M_{3}\left(k_{x}^{2}+k_{y}^{2}\right)+L_{2} k_{z}^{2}
\end{array}\right) .
$$

Again, $\tilde{A}_{(1,2)}, L_{(1,2)}, M_{(1,2,3)}$, and $N_{(1,2,3)}$ are given by:

$$
\begin{gathered}
\tilde{A}_{1}=\frac{1}{2 m_{\|}}-\frac{P_{2}^{2}}{E_{\mathrm{g}}} \text { and } \tilde{A}_{2}=\frac{1}{2 m_{\perp}}-\frac{P_{1}^{2}}{E_{\mathrm{g}}} \\
L_{1}=\frac{1}{2}\left(A_{2}+A_{4}+A_{5}\right)-\frac{P_{1}^{2}}{E_{\mathrm{g}}} \text { and } L_{2}=\frac{1}{2} A_{1}+\frac{P_{2}^{2}}{E_{\mathrm{g}}} \\
M_{1}=\frac{1}{2}\left(A_{2}+A_{4}-A_{5}\right), \quad M_{2}=\frac{1}{2}\left(A_{1}+A_{3}\right), \text { and } M_{3}=\frac{1}{2} A_{2} \\
N_{1}=\frac{1}{2} A_{5}+\frac{P_{2}^{2}}{E_{\mathrm{g}}}, \quad N_{2}=\frac{1}{\sqrt{2}} A_{6}+\frac{P_{1} P_{2}}{E_{\mathrm{g}}} \text { and } N_{3}=i \sqrt{2} A_{7} .
\end{gathered}
$$

From symmetry considerations the $B$ terms are zero. The commonly small parameter $A_{7}$ as well as its counterpart in the upper conduction bands, $A_{7}^{\mathrm{c}}$, were also set to zero. This follows from for $B_{x}:\langle S|\mathbf{k} \cdot \mathbf{p}| i\rangle\langle i|\mathbf{k} \cdot \mathbf{p}| X\rangle=k_{y} k_{z}\left\langle S\left|p_{y}\right| Y\right\rangle\left\langle Y\left|p_{z}\right| X\right\rangle+$ $k_{y} k_{z}\left\langle S\left|p_{z}\right| Z\right\rangle\left\langle Z\left|p_{y}\right| X\right\rangle$, and that a reflection in the $x-z$ plane: $\mathrm{y} \rightarrow-y, \mathrm{x} \rightarrow \mathrm{x}, \mathrm{z} \rightarrow \mathrm{z}$ is a symmetry of WZ such that $\left\langle Y\left|p_{z}\right| X\right\rangle=$ $\left\langle Z\left|p_{y}\right| X\right\rangle=0$. The coupling between the upper and the lower eight bands is realized via:

$$
\Gamma(\mathbf{k})=\left(\begin{array}{cccccccc}
0 & i P_{2}^{\prime} k_{x} & i P_{2}^{\prime} k_{y} & i P_{1}^{\prime} k_{z} & 0 & 0 & 0 & 0 \\
-i P_{2}^{\prime \prime \prime} k_{x} & 0 & -i \Delta_{2}^{c v} & 0 & 0 & 0 & 0 & \Delta_{3,1}^{c v} \\
-i P^{\prime \prime \prime} k_{y} & i \Delta_{2}^{c v} & 0 & 0 & 0 & 0 & 0 & -i \Delta_{3,1}^{c v} \\
-i P_{1}^{\prime \prime \prime} k_{z} & 0 & 0 & 0 & 0 & -\Delta_{3,2}^{c v} & i \Delta_{3,2}^{c v} & 0 \\
0 & 0 & 0 & 0 & 0 & i P_{2}^{\prime \prime \prime} k_{x} & i P_{2}^{\prime \prime \prime} k_{y} & i P_{1}^{\prime \prime \prime} k_{z} \\
0 & 0 & 0 & \Delta_{3,1}^{c v} & -i P_{2}^{\prime} k_{x} & 0^{\prime} & i \Delta_{2}^{c v} & 0 \\
0 & 0 & 0 & -i \Delta_{3,1}^{c v} & -i P_{2}^{\prime} k_{y} & -i \Delta_{2}^{c v} & 0 & 0 \\
0 & \Delta_{3,2}^{c v} & i \Delta_{3,2}^{c v} & 0 & -i P_{1}^{\prime} k_{z} & 0 & 0 & 0
\end{array}\right) .
$$

A schematic view of basis functions and respective couplings is shown in Fig. 8. Note that the small but nonzero coupling of the $\Gamma_{8 \mathrm{c}}$ and the $\Gamma_{9 \mathrm{v}}$ as well as the corresponding $\Gamma_{7 \mathrm{c}}$ and $\Gamma_{9 \mathrm{c}}$ is neglected.

\section{Conditions and initial data for the parameter fitting}

The parameter fitting algorithm has a number of parameters that can be adjusted to achieve a particularly good representation of selected parts of the band structure. For the band structure shown in Fig. 4, these were the following: First, the priority of the top four valence bands has been set to 1000 , and for the bottom four conduction bands to 2000, while the default applied to the other bands is 1 . In the respective output of the fit in Fig. 4, black solid lines indicate those with larger priority whereas the others are depicted in gray. Furthermore, priority was given to the $\mathrm{M}, \Gamma$, and A points and another two points between $\mathrm{M}$ and $\Gamma$ where a Gaussian function was multiplied to Eq. (1) with a peak value of 1000.
The parameter space was mapped onto a Sobol sequence of 10000 elements. With these data, the fitting of all parameters for a band structure with 182 sampling points takes about $3300 \mathrm{~s}$ on a single CPU (Intel Xeon E5, 3.5 GHz).

\section{Ellipticity conditions}

A linear differential operator of second order as it occurs structurally, e.g., as a component of a multiband $\mathbf{k} \cdot \mathbf{p}$ Hamiltonian, that acts on a scalar function $u(\mathbf{x})$ defined as $[15,17]$ :

$$
\left(-\sum_{i, j=1}^{n} \partial_{i} a_{i j}(\mathbf{x}) \partial_{j}+\sum_{i=1}^{n} b_{i}(\mathbf{x}) \partial_{i}+c(\mathbf{x})\right) u(\mathbf{x})
$$




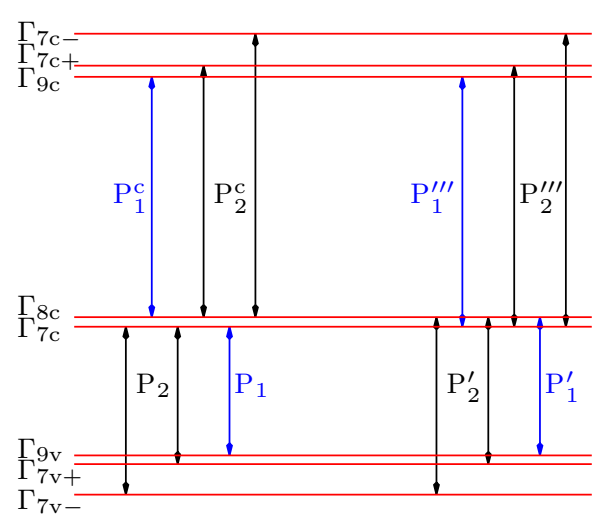

FIG. 8. Term scheme of basis bands and coupling elements.

is called elliptic if the second-order differential operator matrix $\mathbf{K}\left(a_{i j}(\mathbf{k})\right)$ is positive definite [15]. If one then assumes $u$ to be defined inside a region $\Omega$ and zero at its boundary $\partial \Omega$, left multiplying the second-order terms of Eq. (A10) with a test function $v^{\star}$ and integrating over the region yields

$$
\alpha(u, v):=\int_{\Omega} \sum_{i, j=1}^{n} \partial_{i} v^{\star} a_{i j}(\mathbf{x}) \partial_{j} u d \Omega .
$$

For an elliptic operator and a normed function space $V$, this bilinear form is convex:

$$
\alpha(u, v)>C\|u\|_{V} \forall u \in V .
$$

Rewriting Eq. (A11) in matrix form using the differential operator matrix $\mathbf{K}$ we obtain:

$$
\alpha(u, v)=\int_{\Omega}\left(\partial_{x} v^{\star} \partial_{y} v^{\star} \partial_{z} v^{\star}\right) \mathbf{K}\left(\begin{array}{c}
\partial_{x} u \\
\partial_{y} u \\
\partial_{z} u
\end{array}\right) d \Omega .
$$

If $\mathbf{K}$ is positive definite for all $\mathbf{x}, \alpha(\cdot, \cdot)$ will be convex. Within a heterostructure, the coupled differential equation system is defined within an envelope function vector $\mathbf{f}$. The analogy to Eq. (A11) here reads [15]:

$$
\hat{\alpha}(\mathbf{f}, \mathbf{v})=\int_{\Omega} \sum_{i j k l} \partial_{i} v_{k}^{\star} h_{i j}^{k l} \partial_{j} f_{l} d \Omega
$$

with $h_{i j}^{k l}=\left(\mathbf{H}_{i j}^{(2)}\right)_{k l}$ where $k$ and $l$ are the indices of the $m$ Bloch bands involved and $i$ and $j=x, y, z$. For a system quantized in all three directions, the matrix form of Eq. (A14) is given by:

$$
\begin{gathered}
\hat{\alpha}(\mathbf{u}, \mathbf{v})=\int_{\Omega}\left(\partial_{x} v_{1}^{\star} \partial_{y} v_{1}^{\star} \ldots \partial_{y} v_{m}^{\star} \partial_{z} v_{m}^{\star}\right) \\
\times\left(\begin{array}{ccccc}
h_{11}^{11} & h_{12}^{11} & \cdots & h_{12}^{1 m} & h_{13}^{1 m} \\
h_{21}^{11} & h_{22}^{11} & \cdots & h_{22}^{1 m} & h_{23}^{1 m} \\
\vdots & \vdots & \ddots & \vdots & \vdots \\
h_{21}^{m 1} & h_{22}^{m 1} & \cdots & h_{22}^{m m} & h_{23}^{m m} \\
h_{31}^{m 1} & h_{32}^{m 1} & \cdots & h_{32}^{m m} & h_{33}^{m m}
\end{array}\right)\left(\begin{array}{c}
\partial_{x} u_{1} \\
\partial_{y} u_{1} \\
\vdots \\
\partial_{y} u_{m} \\
\partial_{z} u_{m}
\end{array}\right) \partial \Omega
\end{gathered}
$$

and has strictly positive eigenvalues if the matrix defined by $h_{i j}^{k l}$ is positive definite. For Hamiltonians related to the valence band, the available energy spectrum ranges from $-\infty$ to the
TABLE VI. Initial values, search space ranges, and final fitting parameters obtained for WZ GaAs within the 16-band $\mathbf{k} \cdot \mathbf{p}$ model.

\begin{tabular}{lccrr}
\hline \hline Parameter & Initial & Range & Final, $\varepsilon=0$ & $\varepsilon=0.1$ \\
\hline $\mathrm{m}_{\|}^{e}\left(\mathrm{~m}_{0}\right)$ & 0.04 & 0.01 & 0.032 & 0.042 \\
$\mathrm{~m}_{\perp}^{e}\left(\mathrm{~m}_{0}\right)$ & 0.1 & 0.01 & 0.090 & 0.101 \\
$\mathrm{~A}_{1}\left(\hbar^{2} / \mathrm{m}_{0}\right)$ & -5 & 2 & -1.386 & -2.148 \\
$\mathrm{~A}_{2}\left(\hbar^{2} / \mathrm{m}_{0}\right)$ & -0.5 & 0.4 & -0.816 & -0.855 \\
$\mathrm{~A}_{3}\left(\hbar^{2} / \mathrm{m}_{0}\right)$ & 5 & 4 & 0.003 & 1.104 \\
$\mathrm{~A}_{4}\left(\hbar^{2} / \mathrm{m}_{0}\right)$ & 0.5 & 0.4 & 0.159 & 0.007 \\
$\mathrm{~A}_{5}\left(\hbar^{2} / \mathrm{m}_{0}\right)$ & -0.5 & 0.4 & -1.241 & -0.855 \\
$\mathrm{~A}_{6}\left(\hbar^{2} / \mathrm{m}_{0}\right)$ & -0.5 & 0.2 & 0.176 & -0.753 \\
$\mathrm{P}_{1}(\mathrm{eV} \cdot \AA)$ & 1.44 & 0.288 & 1.369 & 0.229 \\
$\mathrm{P}_{2}(\mathrm{eV} \cdot \AA)$ & 1.44 & 0.288 & 2.409 & 1.379 \\
$\mathrm{~m}_{\|}^{c}\left(\mathrm{~m}_{0}\right)$ & 1.0 & 0.2 & 1.117 & 1.378 \\
$\mathrm{~m}_{\perp}^{c}\left(\mathrm{~m}_{0}\right)$ & 0.1 & 0.3 & -0.352 & -0.561 \\
$\mathrm{~A}_{1}^{c}\left(\hbar^{2} / \mathrm{m}_{0}\right)$ & -5 & 2 & -0.951 & -1.361 \\
$\mathrm{~A}_{2}^{c}\left(\hbar^{2} / \mathrm{m}_{0}\right)$ & -0.5 & 0.2 & -0.375 & -0.466 \\
$\mathrm{~A}_{3}^{c}\left(\hbar^{2} / \mathrm{m}_{0}\right)$ & 5 & 2 & 4.772 & 2.374 \\
$\mathrm{~A}_{4}^{c}\left(\hbar^{2} / \mathrm{m}_{0}\right)$ & 5 & 2 & 2.687 & 1.234 \\
$\mathrm{~A}_{5}^{c}\left(\hbar^{2} / \mathrm{m}_{0}\right)$ & -5 & 2 & -4.896 & -1.539 \\
$\mathrm{~A}_{6}^{c}\left(\hbar^{2} / \mathrm{m}_{0}\right)$ & -0.5 & 0.2 & -0.549 & -0.559 \\
$\mathrm{P}_{1}^{c}(\mathrm{eV} \cdot \AA)$ & 1.44 & 3.60 & 0.062 & 0.704 \\
$\mathrm{P}_{2}^{c}(\mathrm{eV} \cdot \AA)$ & 1.44 & 3.60 & 1.342 & -0.017 \\
$\mathrm{P}_{1}^{\prime}(\mathrm{eV} \cdot \AA)$ & 7.2 & 2.88 & 1.494 & 2.570 \\
$\mathrm{P}_{2}^{\prime}(\mathrm{eV} \cdot \AA)$ & 7.2 & 2.88 & 8.033 & 6.847 \\
$\mathrm{P}_{1}^{\prime \prime \prime}(\mathrm{eV} \cdot \AA)$ & 7.2 & 2.88 & 13.939 & 9.421 \\
$\mathrm{P}_{2}^{\prime \prime \prime}(\mathrm{eV} \cdot \AA)$ & 7.2 & 2.88 & 6.132 & 5.490 \\
\hline \hline
\end{tabular}

valence band maximum so that for these cases Eq. (A16) has to be negative definite. For the eight-band model, however, the range of accessible energies ranges from $-\infty$ to $+\infty$ so that no upper or lower boundaries for the eigenvalues exist. Here, the conduction band and valence band parts of the Hamiltonian need to be decoupled so that energy spectra of both band types can be evaluated separately [15].

We have modified the scheme from Ref. [15] to our 16band Hamiltonian as defined in Eq. (A1) above. Following the considerations in Ref. [15], all contributions quadratic in $\mathbf{k}$ enter the Hamiltonian in either the two $6 \times 6$ matrices for the p-like valence and conduction band states or in the $2 \times 2$ matrices for the s-like conduction bands. The s-like conduction band related matrices are furthermore not coupled to each other whereas the two $6 \times 6$ matrices are in fact each two $3 \times 3$ matrices that lack second-order coupling. Correspondingly, the eigenvalues of the valence-band related matrix

$$
h_{i j, \mathrm{v}}^{k l}=\left(\begin{array}{ccccccccc}
L_{1} & 0 & 0 & 0 & N_{1}^{+} & 0 & 0 & 0 & N_{2}^{+} \\
0 & M_{1} & 0 & N_{1}^{-} & 0 & 0 & 0 & 0 & 0 \\
0 & 0 & M_{2} & 0 & 0 & 0 & N_{2}^{-} & 0 & 0 \\
0 & N_{1}^{-} & 0 & M_{1} & 0 & 0 & 0 & 0 & 0 \\
N_{1}^{+} & 0 & 0 & 0 & L_{1} & 0 & 0 & 0 & N_{2}^{+} \\
0 & 0 & 0 & 0 & 0 & M_{2} & 0 & N_{2}^{-} & 0 \\
0 & 0 & N_{2}^{-} & 0 & 0 & 0 & M_{3} & 0 & 0 \\
0 & 0 & 0 & 0 & 0 & N_{2}^{-} & 0 & M_{3} & 0 \\
N_{2}^{+} & 0 & 0 & 0 & N_{2}^{+} & 0 & 0 & 0 & L_{2}
\end{array}\right)
$$


are preferably required to be negative such that

$$
\varrho_{\mathrm{v}}=\left|\frac{\sum_{i, \lambda_{i}>0} \lambda_{i}}{\sum_{j, \lambda_{j}<0} \lambda_{j}}\right|,
$$

which is an estimate for the degree of nonconvexity, is as small as possible and ideally zero. For the five conduction bands in the model, the related matrix reads:

$$
h_{i j, \mathrm{v}}^{k l}=\left(\begin{array}{ccccccccccccccc}
L_{1}^{c} & 0 & 0 & 0 & N_{1}^{c+} & 0 & 0 & 0 & N_{2}^{c+} & 0 & 0 & 0 & 0 & 0 & 0 \\
0 & M_{1}^{c} & 0 & N_{1}^{c-} & 0 & 0 & 0 & 0 & 0 & 0 & 0 & 0 & 0 & 0 & 0 \\
0 & 0 & M_{2}^{c} & 0 & 0 & 0 & N_{2}^{c-} & 0 & 0 & 0 & 0 & 0 & 0 & 0 & 0 \\
0 & N_{1}^{c-} & 0 & M_{1}^{c} & 0 & 0 & 0 & 0 & 0 & 0 & 0 & 0 & 0 & 0 & 0 \\
N_{1}^{c+} & 0 & 0 & 0 & L_{1}^{c} & 0 & 0 & 0 & N_{2}^{c+} & 0 & 0 & 0 & 0 & 0 & 0 \\
0 & 0 & 0 & 0 & 0 & M_{2}^{c} & 0 & N_{2}^{c-} & 0 & 0 & 0 & 0 & 0 & 0 & 0 \\
0 & 0 & N_{2}^{c-} & 0 & 0 & 0 & M_{3}^{c} & 0 & 0 & 0 & 0 & 0 & 0 & 0 & 0 \\
0 & 0 & 0 & 0 & 0 & N_{2}^{c-} & 0 & M_{3}^{c} & 0 & 0 & 0 & 0 & 0 & 0 & 0 \\
N_{2}^{c+} & 0 & 0 & 0 & N_{2}^{c+} & 0 & 0 & 0 & L_{2}^{c} & 0 & 0 & 0 & 0 & 0 & 0 \\
0 & 0 & 0 & 0 & 0 & 0 & 0 & 0 & 0 & A_{1}^{c} & 0 & 0 & 0 & 0 & 0 \\
0 & 0 & 0 & 0 & 0 & 0 & 0 & 0 & 0 & 0 & A_{1}^{c} & 0 & 0 & 0 & 0 \\
0 & 0 & 0 & 0 & 0 & 0 & 0 & 0 & 0 & 0 & 0 & A_{2}^{c} & 0 & 0 & 0 \\
0 & 0 & 0 & 0 & 0 & 0 & 0 & 0 & 0 & 0 & 0 & 0 & A_{1} & 0 & 0 \\
0 & 0 & 0 & 0 & 0 & 0 & 0 & 0 & 0 & 0 & 0 & 0 & 0 & A_{1} & 0 \\
0 & 0 & 0 & 0 & 0 & 0 & 0 & 0 & 0 & 0 & 0 & 0 & 0 & 0 & A_{2}
\end{array}\right)
$$

with its eigenvalues being preferably positive such that

$$
\varrho_{\mathrm{c}}=\left|\frac{\sum_{i, \lambda_{i}<0} \lambda_{i}}{\sum_{j, \lambda_{j}>0} \lambda_{j}}\right| .
$$

Finally, we sum up both conditions to $\varrho=\varrho_{\mathrm{v}}+\varrho_{\mathrm{c}}$ as a total estimate of the degree of nonconvexity. Note that $N_{(1,2)}^{(c) \pm}=N_{(1,2)}^{(c)}$, as a detailed discussion of asymmetric operator ordering as discussed in Refs. [15-17] or a spectral analysis of the Hamiltonian as discussed in Ref. [41] is beyond the scope of the present work. However, our fitting scheme can be modified to identify the best parameters for asymmetric operators as well in a straight-forward manner (Table VI).

[1] A. D. Andreev and E. P. O’Reilly, Phys. Rev. B 62, 15851 (2000).

[2] V. A. Fonoberov and A. A. Balandin, J. Appl. Phys. 94, 7178 (2003).

[3] M. Winkelnkemper, A. Schliwa, and D. Bimberg, Phys. Rev. B 74, 155322 (2006).

[4] O. Marquardt, D. Mourad, S. Schulz, T. Hickel, G. Czycholl, and J. Neugebauer, Phys. Rev. B 78, 235302 (2008).

[5] A. Schliwa, M. Winkelnkemper, A. Lochmann, E. Stock, and D. Bimberg, Phys. Rev. B 80, 161307(R) (2009).

[6] O. Marquardt, L. Geelhaar, and O. Brandt, Nano Lett. 15, 4289 (2015).

[7] I. Vurgaftman, J. R. Meyer, and L. R. Ram-Mohan, J. Appl. Phys. 89, 5815 (2001).

[8] I. Vurgaftman and J. R. Meyer, J. Appl. Phys. 94, 3675 (2003).

[9] P. Rinke, M. Winkelnkemper, A. Qteish, D. Bimberg, J. Neugebauer, and M. Scheffler, Phys. Rev. B 77, 075202 (2008).

[10] S.-H. Wei, S. B. Zhang, and A. Zunger, J. Appl. Phys. 87, 1304 (2000).

[11] S. Z. Karazhanov and L. C. Lew Yan Voon, Semiconductors 39, 161 (2005).

[12] O. Marquardt, M. Ramsteiner, P. Corfdir, L. Geelhaar, and O. Brandt, Phys. Rev. B 95, 245309 (2017).

[13] M. Cardona and F. H. Pollak, Phys. Rev. 142, 530 (1966).
[14] S. Richard, F. Aniel, and G. Fishman, Phys. Rev. B 70, 235204 (2004).

[15] R. G. Veprek, S. Steiger, and B. Witzigmann, Phys. Rev. B 76, 165320 (2007).

[16] R. G. Veprek, S. Steiger, and B. Witzigmann, J. Comput. Electron. 7, 521 (2008).

[17] R. G. Veprek, S. Steiger, and B. Witzigmann, Opt. Quantum Electron. 40, 1169 (2008).

[18] P. Corfdir, B. V. Hattem, E. Uccelli, S. Conesa-Boj, P. Lefebvre, A. F. i Morral, and R. T. Phillips, Nano Lett. 13, 5303 (2013).

[19] P. Corfdir, O. Marquardt, R. B. Lewis, C. Sinito, M. Ramsteiner, A. Trampert, U. Jahn, L. Geelhaar, O. Brandt, and V. M. Fomin, Adv. Mater. 31, 1805645 (2019).

[20] M. I. McMahon and R. J. Nelmes, Phys. Rev. Lett. 95, 215505 (2005).

[21] A. Belabbes, J. Furthmüller, and F. Bechstedt, Phys. Rev. B 87, 035305 (2013).

[22] M. A. Caro, S. Schulz, and E. P. O'Reilly, J. Phys.: Condens. Matter 25, 025803 (2013).

[23] M. A. Caro, S. Schulz, and E. P. O’Reilly, Phys. Rev. B 91, 075203 (2015).

[24] S. L. Chuang and C. S. Chang, Phys. Rev. B 54, 2491 (1996).

[25] S. Steiner, S. Khmelevskyi, M. Marsmann, and G. Kresse, Phys. Rev. B 93, 224425 (2016). 
[26] M. M. Rieger and P. Vogl, Phys. Rev. B 48, 14276 (1993), see erratum [42].

[27] D. J. Dugdale, S. Brand, and R. A. Abram, Phys. Rev. B 61, 12933 (2000).

[28] X. Zhou, F. Bertazzi, M. Goano, G. Ghione, and E. Bellotti, J. Appl. Phys. 116, 033709 (2014).

[29] S. Kucherenko, Application of quasi-monte carlo methods in global optimization, in Global Optimization: From Theory to Implementation, edited by L. Liberti and N. Maculan (Springer US, Boston, 2006), pp. 111-133.

[30] I. M. Sobol, U.S.S.R Comput. Maths. Math. Phys. 7, 86 (1967).

[31] H. Niederreiter, Random Number Generation and Quasi-Monte Carlo Methods (Society for Industrial and Applied Mathematics, Philadelphia, 1992).

[32] T. Cheiwchanchamnangij and W. R. L. Lambrecht, Phys. Rev. B 84, 035203 (2011).
[33] A. Belabbes, C. Panse, J. Furthmüller, and F. Bechstedt, Phys. Rev. B 86, 075208 (2012).

[34] F. Bechstedt and A. Belabbes, J. Phys.: Condens. Matter 25, 273201 (2013).

[35] J. Heyd, G. E. Scuseria, and M. Ernzerhof, J. Chem. Phys. 118, 8207 (2003).

[36] J. P. Perdew, M. Ernzerhof, and K. Burke, J. Chem. Phys. 105, 9982 (1996).

[37] G. Kresse and J. Furthmüller, Phys. Rev. B 54, 11169 (1996).

[38] P. E. Blöchl, Phys. Rev. B 50, 17953 (1994).

[39] G. Kresse and D. Joubert, Phys. Rev. B 59, 1758 (1999).

[40] J. Paier, M. Marsman, K. Hummer, G. Kresse, I. C. Gerber, and J. G. Angyan, J. Chem. Phys. 124, 154709 (2006).

[41] U. Bandelow, H. C. Kaiser, T. Koprucki, and J. Rehberg, Numer. Funct. Anal. Optim. 21, 379 (2000).

[42] M. M. Rieger and P. Vogl, Phys. Rev. B 50, 8138 (1994). 